TRANSACTIONS OF THE

AMERICAN MATHEMATICAL SOCIETY

Volume 348, Number 11, November 1996

\title{
PACKING DIMENSION AND CARTESIAN PRODUCTS
}

\author{
CHRISTOPHER J. BISHOP AND YUVAL PERES
}

\begin{abstract}
We show that for any analytic set $A$ in $\mathbf{R}^{d}$, its packing dimension $\operatorname{dim}_{\mathrm{P}}(A)$ can be represented as $\sup _{B}\left\{\operatorname{dim}_{\mathrm{H}}(A \times B)-\operatorname{dim}_{\mathrm{H}}(B)\right\}$, where the supremum is over all compact $\operatorname{sets} B$ in $\mathbf{R}^{d}$, and $\operatorname{dim}_{\mathrm{H}}$ denotes Hausdorff dimension. (The lower bound on packing dimension was proved by Tricot in 1982.) Moreover, the supremum above is attained, at least if $\operatorname{dim}_{\mathrm{P}}(A)<d$. In contrast, we show that the dual quantity $\inf _{B}\left\{\operatorname{dim}_{\mathrm{P}}(A \times B)-\operatorname{dim}_{\mathrm{P}}(B)\right\}$, is at least the "lower packing dimension" of $A$, but can be strictly greater. (The lower packing dimension is greater than or equal to the Hausdorff dimension.)
\end{abstract}

\section{INTRODUCTION}

Marstrand's product theorem ([9]) asserts that if $A, B \subset \mathbf{R}^{d}$ then

$$
\operatorname{dim}_{\mathrm{H}}(A \times B) \geq \operatorname{dim}_{\mathrm{H}}(A)+\operatorname{dim}_{\mathrm{H}}(B),
$$

where "dim ${ }_{H}$ " denotes Hausdorff dimension. Refining earlier results of Besicovitch and Moran [3], Tricot [14] showed that

$$
\operatorname{dim}_{\mathrm{H}}(A \times B)-\operatorname{dim}_{\mathrm{H}}(B) \leq \operatorname{dim}_{\mathrm{P}}(A),
$$

where "dim ${ }_{\mathrm{P}}$ " denotes packing dimension (see the next section for background). Tricot [15] and also Hu and Taylor [6] asked if this is sharp, i.e., given $A$, can the right-hand side of (1) be approximated arbitrarily well by appropriate choices of $B$ on the left-hand side? Our first result, proved in Section 3, states that this is possible.

Theorem 1.1. For any analytic set $A$ in $\mathbf{R}^{d}$

$$
\sup _{B}\left\{\operatorname{dim}_{\mathrm{H}}(A \times B)-\operatorname{dim}_{\mathrm{H}}(B)\right\}=\operatorname{dim}_{\mathrm{P}}(A),
$$

where the supremum is over all compact sets $B \subset \mathbf{R}^{d}$.

The proof actually shows that, when $\operatorname{dim}_{\mathrm{P}}(A)<d$, the supremum is attained at some compact set $B$ of Hausdorff dimension $d-\operatorname{dim}_{\mathrm{P}}(A)$. We remark that there are compact sets $A \subset \mathbf{R}^{d}$ for which the supremum is attained only with sets $B$ of dimension $d-\operatorname{dim}_{\mathrm{P}}(A)$.

Received by the editors April 27, 1995 .

1991 Mathematics Subject Classification. Primary 28A80.

Key words and phrases. Hausdorff dimension, packing dimension, Cartesian product, tree.

Supported in part by NSF grant \# DMS 9204092 and by an Alfred P. Sloan Foundation Fellowship.

Research partially supported by NSF grant \# DMS-9404391.

(C)1996 American Mathematical Society 
The sets which have equal Hausdorff and packing dimensions have been singled out by Tricot [14] (who called them "dimension-regular") and by Taylor [12] because of their good behavior in Cartesian products and probabilistic applications. Theorem 1.1 shows that these are the only compact sets which have these good properties universally.

A related question concerns the behavior of packing dimension under products. Tricot [14] showed that

$$
\operatorname{dim}_{\mathrm{P}}(E \times F) \geq \operatorname{dim}_{\mathrm{H}}(E)+\operatorname{dim}_{\mathrm{P}}(F) .
$$

$\mathrm{Hu}$ and Taylor [6] asked if all sets $E \subset \mathbf{R}$ satisfy

$$
\inf _{F}\left\{\operatorname{dim}_{\mathrm{P}}(E \times F)-\operatorname{dim}_{\mathrm{P}}(F)\right\}=\operatorname{dim}_{\mathrm{H}}(E) ?
$$

The answer turns out to be more delicate. For a bounded set $E$ in a metric space, denote by $N(E, \epsilon)$ the maximal cardinality of a collection of disjoint closed balls of radius $\epsilon$ with centers in $E$. Let

$$
\underline{\operatorname{dim}}_{\mathrm{M}}(E)=\liminf _{\epsilon \rightarrow 0} \frac{\log N(E, \epsilon)}{|\log \epsilon|}
$$

be the lower Minkowski dimension of E. Following Mattila [10], we call the "regularization" of this index the lower packing dimension, denoted $\underline{\operatorname{dim}}_{\mathrm{P}}$, although it is not constructed using packing measures. It is defined, for any set $E$ in a metric space, by

$$
\underline{\operatorname{dim}}_{\mathrm{P}}(E)=\inf _{E \subset \cup_{j} E_{j}} \sup _{j} \underline{\operatorname{dim}}_{\mathrm{M}}\left(E_{j}\right),
$$

where the infimum is over all countable collections of bounded sets $\left\{E_{j}\right\}$ whose union contains $E$.

It is easy to see that $\operatorname{dim}_{\mathrm{H}}(E) \leq \operatorname{dim}_{\mathrm{P}}(E)$ for any set $E$. A set $E$ where the inequality is strict was constructed by Tricot [14]; A simpler example, due to B. Weiss, was described in Benjamini and Peres ([1] , pp. 587).

The following proposition is proved in Section 4 .

Proposition 1.2. For any compact set $E$ in $\mathbf{R}^{d}$,

$$
\inf _{F}\left\{\operatorname{dim}_{\mathrm{P}}(E \times F)-\operatorname{dim}_{\mathrm{P}}(F)\right\} \geq \underline{\operatorname{dim}}_{\mathrm{P}}(E)
$$

where the infimum is over all compact sets $F \subset \mathbf{R}^{d}$. There exist compact sets $E \subset \mathbf{R}$ for which the inequality in (7) is strict.

\section{BACKGROUND ON PACKING DIMENSION AND TREES}

For background on Hausdorff measures and dimension we refer to Falconer [4]. A more naive notion is the upper Minkowski dimension (sometimes called the "upper box dimension") defined for any set $E$ in a metric space by

$$
\overline{\operatorname{dim}}_{\mathrm{M}}(E)=\limsup _{\epsilon \rightarrow 0} \frac{\log N(E, \epsilon)}{|\log \epsilon|} .
$$

(The lower and upper Minkowski dimensions are infinite if $E$ is not totally bounded.) Tricot [13], [14] introduced packing dimension, which plays a dual role to Hausdorff dimension in many settings. For our present purpose, the representation of 
packing dimension which is convenient to use as a definition, is as a regularization of upper Minkowski dimension:

$$
\operatorname{dim}_{\mathrm{P}}(A)=\inf _{A \subset \cup_{j} A_{j}} \sup _{j} \overline{\operatorname{dim}}_{\mathrm{M}}\left(A_{j}\right),
$$

where the infimum is over all countable covers of $A$. (See Tricot [14], Proposition 2, or Falconer [4], Proposition 3.8.)

Part ( $i$ ) of the next lemma is due to Tricot [14] (see also Falconer [4]); Part (ii) for trees can be found in Benjamini and Peres ([2], Proposition 4.2(b)); the general version given is in Falconer and Howroyd [5] and in Mattila and Mauldin [11].

Lemma 2.1. (i) Let $E$ be a closed set in a complete metric space. If any open set $V$ which intersects $E$ satisfies $\overline{\operatorname{dim}}_{\mathrm{M}}(E \cap V) \geq \alpha$, then $\operatorname{dim}_{\mathrm{P}}(E) \geq \alpha$.

(ii) Let $E$ be a subset of a separable metric space, with $\operatorname{dim}_{\mathrm{P}}(E)>\alpha$. Then there is a (relatively closed) nonempty subset $\widetilde{E}$ of $E$, such that $\operatorname{dim}_{\mathrm{P}}(\widetilde{E} \cap V)>\alpha$ for any open set $V$ which intersects $\widetilde{E}$.

(iii) The analogues of (i) and (ii) with $\underline{\operatorname{dim}}_{\mathrm{M}}$ and $\underline{\operatorname{dim}}_{\mathrm{P}}$ in place of $\overline{\operatorname{dim}}_{\mathrm{M}}$ and $\operatorname{dim}_{\mathrm{P}}$ respectively, are valid.

Proof. (i) See Tricot [14] or Falconer [4], Proposition 3.6. (ii) Define $\widetilde{E}$ to be the set of points $x \in E$ such that every neighborhood $W$ of $x$ satisfies $\operatorname{dim}_{\mathrm{P}}(E \cap W)>\alpha$. Clearly $\widetilde{E}$ is relatively closed in $E$. If $\widetilde{E}$ was empty, then $E$ would be covered by relatively open sets $E \cap W$ of packing dimension at most $\alpha$. Passing to a countable subcover (using separability) would yield a contradiction. To verify that $\widetilde{E}$ has the required property, let $V$ be any open set which intersects $\widetilde{E}$. By the definition of $\widetilde{E}$, the intersection $V \cap(E \backslash \widetilde{E})$ is covered by the union of the open sets $W$ such that $\operatorname{dim}_{\mathrm{P}}(E \cap W) \leq \alpha$, and using separability again,

$$
\alpha<\operatorname{dim}_{\mathrm{P}}(E \cap V) \leq \max \left\{\operatorname{dim}_{\mathrm{P}}(\widetilde{E} \cap V), \alpha\right\} .
$$

This completes the proof.

(iii) The proofs of the previous two parts transfer.

Tree Notation. We will represent closed subsets of the unit cube by infinite trees, and use finite trees as tools in our constructions. A (rooted) tree $T$ is a connected acyclic graph with a distinguished vertex $\rho$ designated as the root. It may be finite or infinite, but every vertex must have finite degree. For every vertex $v$ we write $|v|$ for its level, i.e., the number of edges between it and the root. Neighbors of $v$ at level $|v|+1$ are called children of $v$. More generally, if $v$ is on the path from the root to a vertex $w$, then $w$ is called a descendant of $v$, and $v$ is an ancestor of $w$. If a vertex of $T$ has no children, it is called a leaf of $T$. If all the leaves of a finite tree $T$ are at level $n$, we say that $T$ has uniform depth $n$. A function $\mu$ on the vertices of $T$ that satisfies Kirchhoff's law

$$
\mu(v)=\sum\{\mu(w): w \text { a child of } v\}
$$

for every vertex $v$ of $T$ which is not a leaf, is called a flow on $T$.

Now we focus on infinite trees without leaves. An infinite self-avoiding path in a tree $T$, starting at the root of $T$, is called a ray. The set of all rays is called the boundary of $T$ and denoted $\partial T$. For two distinct rays $x, y \in \partial T$ we define their distance to be

$$
\operatorname{dist}(x, y)=2^{-n} \text { if } x \text { and } y \text { have exactly } n \text { edges in common. }
$$




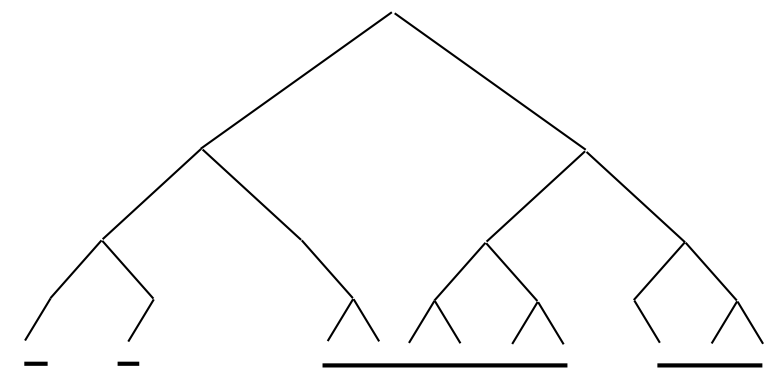

Figure 1. A set $K$ and the initial levels of an associated tree $T(K)$.

This makes $\partial T$ a compact metric space. For any vertex $v$, denote by $[v]$ the set of rays going through $v$. There is a one-to-one correspondence between measures on $\partial T$ and flows on $T$ (where the $\mu$-measure of the set $[v]$ is $\mu(v)$ ), since the additivity axiom for measures corresponds to Kirchhoff's law (10) for flows. Probability measures correspond to unit flows. A set of vertices $\Pi$ that intersects every ray of $T$ is called a cut-set. Equivalently, $\Pi$ is a cut-set iff $\{[v]: v \in \Pi\}$ is a cover of $\partial T$.

The closed dyadic subcubes of the unit cube in $\mathbf{R}^{d}$ have a natural tree structure when ordered by inclusion, and the resulting tree is regular with $2^{d}$ children to every vertex; we denote this tree by $\Gamma^{\left(2^{d}\right)}$.

We employ the canonical mapping $\mathcal{R}$ from the boundary of $\Gamma^{\left(2^{d}\right)}$ to the cube $[0,1]^{d}$. Formally, label the edges from each vertex of $\Gamma^{\left(2^{d}\right)}$ to its children in a one-to-one manner with the vectors in $\Omega=\{0,1\}^{d}$. Then the boundary $\partial \Gamma^{\left(2^{d}\right)}$ is identified with the sequence space $\Omega^{\mathbf{Z}^{+}}$and we define the binary representation $\operatorname{map} \mathcal{R}: \Omega^{\mathbf{Z}^{+}} \rightarrow[0,1]^{d}$ by

$$
\mathcal{R}\left(\omega_{1}, \omega_{2}, \ldots\right)=\sum_{n=1}^{\infty} 2^{-n} \omega_{n} .
$$

Similarly, a vertex $v$ at level $k$ of $\Gamma^{\left(2^{d}\right)}$ is identified with a finite sequence $\left(\omega_{1}, \ldots, \omega_{k}\right)$ $\in \Omega^{k}$ and we write $\mathcal{R}(v)$ for the cube of side $2^{-k}$ obtained as the image under $\mathcal{R}$ of all sequences in $\Omega^{\mathbf{Z}^{+}}$with prefix $\left(\omega_{1}, \ldots, \omega_{k}\right)$.

With the notation above, let $T$ be an infinite subtree of the regular $2^{d}$-ary tree $\Gamma^{\left(2^{d}\right)}$, with the same root and without leaves. We may identify $\partial T$ with a closed subset of $\Omega^{\mathbf{Z}^{+}}$. The image of this set under $\mathcal{R}$ is a compact set $K \subset[0,1]^{d}$. We say that $T$ is associated with $K$ and denote it by $T(K)$ even though it is sometimes not uniquely determined by $K$. (See Figure 1.) (For instance, the one-point set $\{1 / 2\}$ has three subtrees of $\Gamma^{\left(2^{d}\right)}$ associated with it, two of them consisting of single rays and the other consisting of two disjoint rays.)

Given any compact set $K \subset[0,1]^{d}$, the dyadic cubes which intersect $K$ form a subtree of $\Gamma^{\left(2^{d}\right)}$, and this is a natural choice for $T(K)$, but not all leafless subtrees of $\Gamma^{\left(2^{d}\right)}$ can arise in this way.

Given a closed set $K \subset[0,1]^{d}$, and an associated tree $T=T(K)$, let $\left|T_{n}(K)\right|$ denote the number of vertices of the tree $T(K)$ at level $n$.

Every closed dyadic cube intersecting $K$ is either the image under $\mathcal{R}$ of a vertex in $T$ or adjacent to such an image cube of the same size. Therefore, the number of closed dyadic cubes of side length $2^{-n}$ which intersect $K$ is at least $\left|T_{n}(K)\right|$ and 
at most $3^{d} \cdot\left|T_{n}(K)\right|$. It is a standard fact that the upper and lower Minkowski dimensions can be calculated by counting dyadic cubes; comparing this with the the definition (11) of the metric on $\partial T$ gives

$$
\overline{\operatorname{dim}}_{\mathrm{M}}(K)=\limsup _{n \rightarrow \infty} \frac{1}{n} \log _{2}\left|T_{n}(K)\right|=\overline{\operatorname{dim}}_{\mathrm{M}}(\partial T(K))
$$

and

$$
\underline{\operatorname{dim}}_{\mathrm{M}}(K)=\liminf _{n \rightarrow \infty} \frac{1}{n} \log _{2}\left|T_{n}(K)\right|=\underline{\operatorname{dim}}_{\mathrm{M}}(\partial T(K)) .
$$

The $\beta$-dimensional Hausdorff content of a set $E$ is

$$
\mathcal{H}_{\infty}^{\beta}(E)=\inf \left\{\sum_{j}\left(\operatorname{diam} E_{j}\right)^{\beta} \mid E \subset \bigcup_{j} E_{j}\right\},
$$

the infimum being over all countable covers of $E$. The Hausdorff dimension of $E$ is

$$
\operatorname{dim}_{\mathrm{H}}(E)=\inf \left\{\beta>0 \mid \mathcal{H}_{\infty}^{\beta}(E)=0\right\} .
$$

For purposes of computing the Hausdorff dimension, it suffices to estimate the Hausdorff content using coverings by dyadic cubes. Such a covering corresponds to a cut-set $\Pi$ of the tree $T(K)$ and we get

$$
\operatorname{dim}_{\mathrm{H}}(K)=\inf \left\{\beta \mid \inf _{\Pi} \sum_{v \in \Pi} 2^{-\beta|v|}=0\right\} .
$$

Call the inner sum a " $\beta$-dimensional cut-set sum." To verify (13), observe the preimage under $\mathcal{R}$ of a closed dyadic cube of side-length $2^{-j}$ is covered by at most $3^{d}$ sets of the form $[v]$ with $v$ at level $j$ of $\Gamma^{\left(2^{d}\right)}$.

The mass distribution principle (see Proposition 4.2 in Falconer ([4]) says that if $K$ supports a positive measure $\mu^{\prime}$ satisfying a Hölder estimate $\mu^{\prime}(F) \leq C(\operatorname{diam} F)^{\beta}$ for any set $F$ and some constant $C$, then $\operatorname{dim}_{\mathrm{H}}(K) \geq \beta$. It suffices to assume the Hölder estimate for dyadic cubes. In terms of the tree, this estimate means that $T(K)$ supports a flow $\mu$ so that the flow going through any vertex $v$ satisfies $\mu(v) \leq C 2^{-\beta|v|}$.

The product tree. Given two compact sets $A, B$ in $[0,1]^{d}$, we can construct a tree associated to the Cartesian product $A \times B \subset[0,1]^{2 d}$ from trees $T(A)$ and $T(B)$. In general, given two trees $T^{1}, T^{2}$, the product tree $T^{1} * T^{2}$ has the vertex set

$$
\left\{\left(v_{1}, v_{2}\right): v_{i} \in T^{i} \text { for } i=1,2 \text { and }\left|v_{1}\right|=\left|v_{2}\right|\right\},
$$

the root $\left(\operatorname{root}\left(T^{1}\right), \operatorname{root}\left(T^{2}\right)\right)$, and the adjacency relation:

the vertex $\left(v_{1}, v_{2}\right)$ is adjacent to $\left(u_{1}, u_{2}\right)$ iff $v_{i}$ is adjacent to $u_{i}$ for $i=1,2$.

It is easy to see that $T(A) * T(B)$ is associated with $A \times B$.

\section{Proof of Theorem 1.1}

Given an analytic set $A$ in $\mathbf{R}^{d}$, denote $\alpha=\operatorname{dim}_{\mathrm{P}}(A)$. Clearly, we may assume that $\alpha>0$; we will first prove the theorem under the further assumption $\alpha<d$. We will construct a compact set $B$ in $\mathbf{R}^{d}$ of Hausdorff dimension $d-\alpha$ so that $\operatorname{dim}_{\mathrm{H}}(A \times B)=d$.

Let $0<\widetilde{\alpha}<\alpha$. Recent results of Joyce and Preiss [7] imply that $A$ contains a subset $A_{0}$ which is closed in $\mathbf{R}^{d}$ with $\operatorname{dim}_{\mathrm{P}}\left(A_{0}\right)>\widetilde{\alpha}$. By partitioning and translating if necessary, we may assume that $A_{0}$ is contained in the unit cube. 


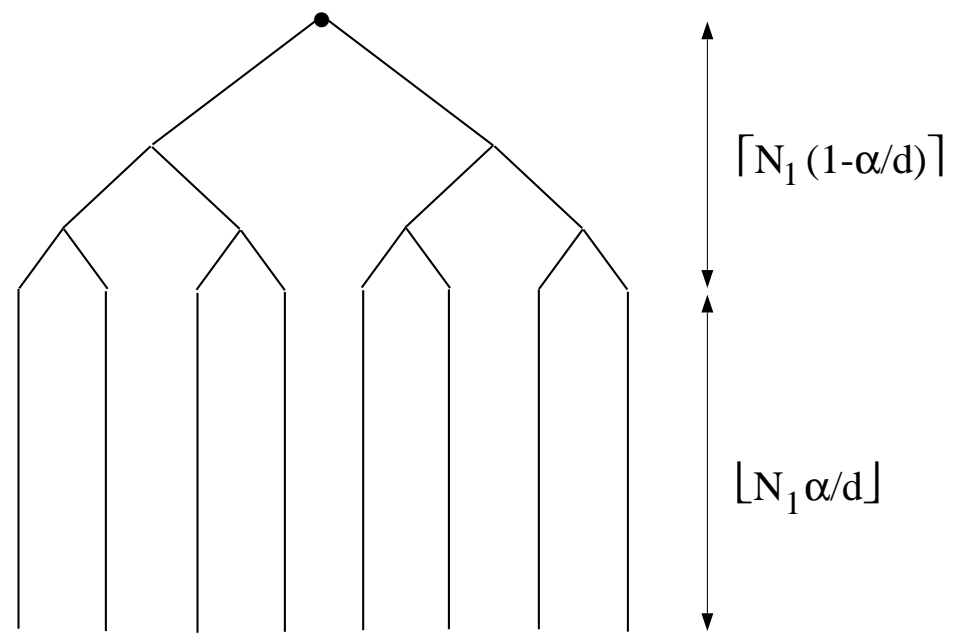

FiguRE 2. Maximal branching followed by no branching

Applying Lemma 2.1(ii) to the boundary of the tree $\partial T\left(A_{0}\right)$, we obtain a subtree $T(\widetilde{A})$ with $\widetilde{A} \subset A_{0}$ such that any open set $V$ on the boundary of this subtree has packing dimension greater than $\widetilde{\alpha}$. This implies that for any vertex $v$ of the tree $T(\widetilde{A})$, we have

$$
\limsup _{n \rightarrow \infty} \frac{1}{n} \log _{2}\left|T_{n}(\widetilde{A}, v)\right| \geq \widetilde{\alpha}
$$

where $\left|T_{n}(\widetilde{A}, v)\right|$ is the number of descendants $w$ of $v$ such that $|w|=n$.

We will consider truncations $T^{k}(\widetilde{A})$ of the tree $T(\widetilde{A})$ to certain levels $N_{k}$, and use them to inductively construct a nested increasing sequence of trees $\Gamma^{k}$, whose limit $\Gamma$ is associated to an approximation $\widetilde{B}$ of the promised set $B$. Simultaneously, we will describe directed unit flows from the root to the boundaries of the product trees $T^{k}(\widetilde{A}) * \Gamma^{k}$ which satisfy uniform Hölder estimates of order $d_{k}$ close to $d$. This will suffice to establish the variational principle (2). A further limiting argument will be needed to show that the supremum there is attained.

First step. By (14), there is a level $N_{1}$ such that $T(\widetilde{A})$ has more than $2^{\widetilde{\alpha} N_{1}}$ vertices at level $N_{1}$. Construct $\Gamma^{1}$ of uniform depth $N_{1}$, where every vertex in the first $\left\lceil N_{1}(1-\alpha / d)\right\rceil$ level has $2^{d}$ children, and every vertex in the remaining $\left\lfloor N_{1} \alpha / d\right\rfloor$ levels has a single child (see Figure 2). The leaves of $\Gamma^{1}$ form a cut-set $\Pi_{1}$ of cardinality at most $2^{N_{1}(d-\alpha)+d}$, so the corresponding $d-\alpha$ dimensional cut-set sum satisfies

$$
\sum_{v \in \Pi_{1}} 2^{(\alpha-d)|v|} \leq 2^{d}
$$

Now consider the uniform unit flow $\mu$ on the product tree $T^{1}(\widetilde{A}) * \Gamma^{1}$ which assigns each vertex at level $N_{1}$ the same mass.

Let $(v, w)$ be a vertex at level $j \leq N_{1}$ of this product tree. Observe that $v$ has at most $2^{d\left(N_{1}-j\right)}$ descendants at level $N_{1}$ of $T(\widetilde{A})$. If $j>\left\lceil N_{1}(1-\alpha / d)\right\rceil$ then $w$ 
has only one descendant at level $N_{1}$ of $\Gamma^{1}$, so that

$$
\mu(v, w) \leq 2^{d\left(N_{1}-j\right)} \cdot 1 \cdot 2^{-\widetilde{\alpha} N_{1}} \cdot 2^{-d N_{1}(1-\alpha / d)}=2^{-d j+(\alpha-\widetilde{\alpha}) N_{1}} .
$$

Denoting

$$
\widetilde{d}:=d-\frac{\alpha-\widetilde{\alpha}}{1-\alpha / d},
$$

we deduce from (15) that the Hölder estimate

$$
\mu(v, w) \leq 2^{-\widetilde{d}|(v, w)|}
$$

holds if $j=|(v, w)|>\left\lceil N_{1}(1-\alpha / d)\right\rceil$. On the other hand, an improved Hölder estimate (with $d$ in place of $\widetilde{d}$ ) clearly holds for smaller values of $j$, since $\Gamma_{1}$ has full branching at these levels. We conclude that (17) is valid for all vertices $(v, w)$ of $T^{1}(\widetilde{A}) * \Gamma^{1}$. For use in the induction below, we define $N_{0}=0$ and $d_{0}=d_{1}=\widetilde{d}$.

The inductive step. We assume that we have constructed a tree $\Gamma^{k}$ of uniform depth $N_{k}$, in which every vertex has at most $2^{d}$ children. Also, we assume there is a unit flow $\mu$ on the product tree $T^{k}(\widetilde{A}) * \Gamma^{k}$ which satisfies $\mu(v, w) \leq 2^{-d_{k-1} j}$ for any $N_{k-1} \leq j \leq N_{k}$ and any vertex $(v, w)$ at level $j$ of the product tree; for leaves $(v, w)$ of $\Gamma^{k}$, we assume the estimate $\mu(v, w) \leq 2^{-d_{k} N_{k}}$ holds.

The construction of $\Gamma^{k+1}$ from $\Gamma^{k}$ is done in four stages:

1. To each leaf of $\Gamma^{k}$ attach a full $2^{d}$-ary tree of depth $k N_{k}$ to obtain a tree $\Gamma^{k, 1}$ of uniform depth $(k+1) N_{k}$.

2. To each leaf of $\Gamma^{k, 1}$ attach a copy of $T^{k}(\widetilde{A})$. This yields a tree $\Gamma^{k, 2}$ of uniform depth $(k+2) N_{k}$, where each leaf of $\Gamma^{k, 2}$ corresponds to some leaf of $T^{k}(\widetilde{A})$ (this is a many-to-one correspondence).

3. Now we mimic the first step of the induction. For each leaf $u$ of $T^{k}(\widetilde{A})$, choose an integer $n(u)$ such that $u$ has at least $2^{n(u) \widetilde{\alpha}}$ descendants at level $|u|+n(u)$, and $n(u) \geq\left(k^{2}+k+1\right) N_{k}$. This is possible by (14). For future reference, we denote by $T^{k, 3}$ the subtree of $T(\widetilde{A})$ obtained by appending to every vertex $u$ at level $N_{k}$ its descendants in the next $n(u)$ levels. To each vertex of $\Gamma^{k, 2}$ corresponding to $u$, append a tree of uniform depth $m(u)=n(u)-(k+1) N_{k}$, where every vertex in the first $\lceil m(u)(1-\alpha / d)\rceil$ levels has $2^{d}$ children, and every vertex in the remaining $\lfloor m(u) \alpha / d\rfloor$ levels has a single child. This defines $\Gamma^{k, 3}$. The leaves of $\Gamma^{k, 3}$ form a cut-set $\Pi_{k+1}$ such that the corresponding $\beta$-dimensional cut-set sum satisfies

$$
\sum_{w \in \Pi_{k+1}} 2^{-\beta|w|} \leq 2^{d(k+1) N_{k}} \cdot \sum_{u \in \partial T^{k}(\widetilde{A})} 2^{m(u)(d-\alpha)+d} \cdot 2^{-\beta\left(N_{k}+n(u)\right)},
$$

where the first factor $2^{d(k+1) N_{k}}$ is a bound on the number of leaves of $\Gamma^{k, 1}$. The number of leaves of $T^{k}(\widetilde{A})$ is at most $2^{d N_{k}}$. Using this and the inequality $m(u) \leq n(u)$, we see that the cut-set sum (18) is bounded by

$$
2^{d(k+1) N_{k}} \cdot \sum_{u \in \partial T^{k}(\widetilde{A})} 2^{d} \cdot 2^{n(u)(d-\alpha-\beta)} \leq 2^{d(k+2) N_{k}+d} \cdot 2^{k^{2} N_{k}(d-\alpha-\beta)} .
$$

where the last inequality used $n(u)>k^{2} N_{k}$. For any fixed $\beta>d-\alpha$, these bounds tend to zero as $k \rightarrow \infty$. 


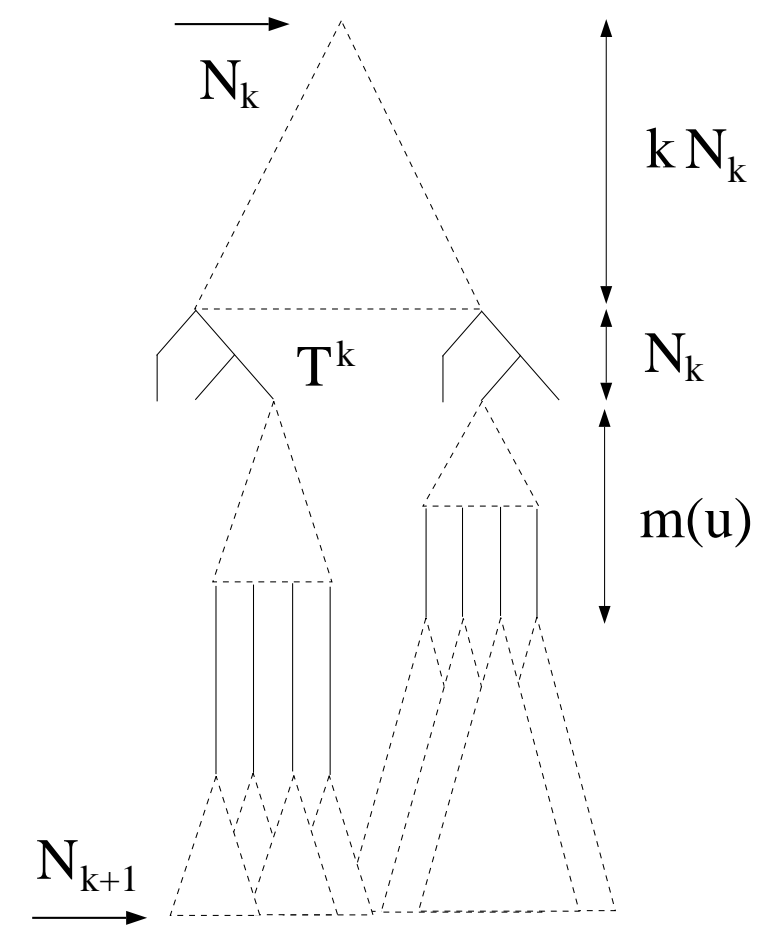

Figure 3. The four stages of the construction

4. Finally, denote by $N_{k+1}$ the maximum depth of $\Gamma^{k, 3}$, and to each leaf $w$ of $\Gamma^{k, 3}$ append a tree of uniform depth $N_{k+1}-|w|$ with $2^{d}$ children at every level. This yields $\Gamma^{k+1}$.

The construction is illustrated in Figure 3. The dashed boxes represent subtrees with full growth $\left(2^{d}\right.$ children at each vertex).

Now that we have constructed the tree $\Gamma^{k+1}$ we want to construct a flow $\mu$ on the product tree $T^{k+1}(\widetilde{A}) * \Gamma^{k+1}$ which extends the flow $\mu$ constructed previously on $T^{k}(\widetilde{A}) * \Gamma^{k}$. (Rather than call the flow constructed at each step $\mu_{k}$, we simply refer to them all as $\mu$ since the definitions are consistent.) It is most convenient to do this in four stages following the construction of the tree.

1. Suppose $(v, w)$ is a leaf of $T^{k}(\widetilde{A}) * \Gamma^{k}$. By our induction hypothesis the flow satisfies the Hölder estimate

$$
\mu(v, w) \leq 2^{-d_{k} N_{k}} .
$$

Let $T^{k, 1}$ be the truncation of $T(\widetilde{A})$ to level $(k+1) N_{k}$ (so it has the same depth as $\left.\Gamma^{k, 1}\right)$. We define the flow on $T^{k, 1} * \Gamma^{k, 1}$ by dividing the mass assigned by $\mu$ to each vertex of the smaller tree $T^{k}(\widetilde{A}) * \Gamma^{k}$ equally among its descendants at level $(k+1) N_{k}$ of the extended tree $T^{k, 1} * \Gamma^{k, 1}$. Since $\Gamma^{k, 1}$ has full branching at these levels (i.e., each vertex has $2^{d}$ children) it is clear that the resulting flow satisfies

$$
\mu(v, w) \leq 2^{-d_{k} j},
$$

for vertices in level $j$ such that $N_{k} \leq j \leq(k+1) N_{k}$. 
2. This is the only stage where we do not divide the mass equally among descendants. Let $T^{k, 2}$ be the truncation of $T(\widetilde{A})$ to level $(k+2) N_{k}$ (so it has the same depth as $\left.\Gamma^{k, 2}\right)$. For each leaf $(v, w)$ of $T^{k, 1} * \Gamma^{k, 1}$, let $u$ be the leaf of $T^{k}(\widetilde{A})$ which is an ancestor of $v$. Divide the mass of $(v, w)$ evenly among the leaves $\left(v^{\prime}, w^{\prime}\right)$ of $T^{k, 2} * \Gamma^{k, 2}$ which satisfy: $v^{\prime}$ is a descendant of $v$ and $w^{\prime}$ is the unique descendant of $w$ corresponding to $u$. At worst, we have concentrated all the mass of $(v, w)$ onto a single descendant, so for leaves $\left(v^{\prime}, w^{\prime}\right)$ of $T^{k, 2} * \Gamma^{k, 2}$ we get

$$
\mu\left(v^{\prime}, w^{\prime}\right) \leq \mu(v, w) \leq 2^{-d k N_{k}} \leq 2^{-d_{k+1}(k+2) N_{k}},
$$

where we define

$$
d_{k+1}:=\min \left\{\frac{d k}{k+2}, \widetilde{d}\right\} .
$$

Thus at level $(k+2) N_{k}$, the flow $\mu$ satisfies a Hölder estimate of order $d_{k+1}$.

3. Next, we extend the definition of $\mu$ to the product tree $T^{k, 3} * \Gamma^{k, 3}$. The two trees in the product do not have uniform depth. Let $v$ be a leaf of $T^{k, 2}$ which is a descendant of a leaf $u$ of $T^{k}$, and let $w$ be a leaf of $\Gamma^{k, 2}$ which corresponds to $u$. Divide the mass $\mu(v, w)$ equally among the descendants of $(v, w)$ at level $N_{k}+n(u)$. Since the flow $\mu$ is Hölder of order $d_{k+1} \leq \tilde{d}$ at $(v, w)$, the argument in step 1 of the induction implies that the same estimate holds for all descendants of $(v, w)$ down to level $N_{k}+n(u)$.

4. Finally, to define the flow on $T^{k+1} * \Gamma^{k+1}$, use equal division again; since $\Gamma^{k+1}$ has full branching here, the Hölder estimate of order $d_{k+1}$ established at the previous stage persists.

This completes the inductive step.

The limit of the trees $\Gamma^{k}$ is a tree $\Gamma$ which is associated to a compact set $\widetilde{B}$ in the cube $[0,1]^{d}$. Each cut-set of $\Gamma$ corresponds to a cover of $\widetilde{B}$ by dyadic cubes, so the cut-set sum estimates (19) in stage 3 of the inductive step imply that

$$
\operatorname{dim}_{\mathrm{H}}(\widetilde{B}) \leq d-\alpha .
$$

The flow $\mu$ extends to the product tree $T(\widetilde{A}) * \Gamma$ and corresponds to a measure $\mu^{\prime}$ on $\widetilde{A} \times B$ which satisfies the estimate $\mu^{\prime}(Q) \leq \operatorname{diam}(Q)^{d_{k}}$ for any dyadic cube $Q$ of side-length at most $2^{-N_{k}}$. Since $d_{k}=\widetilde{d}$ for all large $k$ by (20), the mass distribution principle (see, e.g., Proposition 4.2 in Falconer [4]) implies that

$$
\operatorname{dim}_{\mathrm{H}}(\widetilde{A} \times \widetilde{B}) \geq \widetilde{d}
$$

Under our standing assumption $\alpha=\operatorname{dim}_{\mathrm{P}}(A)<d$, we can get $\widetilde{d}$ arbitrarily close to $d$ by choosing $\widetilde{\alpha}$ close to $\alpha$ and using (16). Thus (21) and (22), combined with Tricot's upper bound (1), prove the variational formula (2) in this case.

Now we show that the supremum in (2) is attained when $\alpha<d$. For each integer $m$, taking $\widetilde{\alpha} \geq \alpha-1 / m$, the construction above yields a compact set $\widetilde{B}_{m}$ in $\mathbf{R}^{d}$ such that

$$
\operatorname{dim}_{\mathrm{H}}\left(\widetilde{B}_{m}\right) \leq d-\alpha \text { and } \operatorname{dim}_{\mathrm{H}}\left(A \times \widetilde{B}_{m}\right) \geq \widetilde{d}_{m}=d-\frac{d}{m(d-\alpha)} .
$$

By translating and scaling, we may assume that each $\widetilde{B}_{m}$ contains the origin and has diameter at most $1 / m$. The union $B:=\bigcup_{m=1}^{\infty} \widetilde{B}_{m}$ is compact, and (23) implies 
that

$$
\operatorname{dim}_{\mathrm{H}}(B) \leq d-\alpha \text { and } \operatorname{dim}_{\mathrm{H}}(A \times B) \geq d
$$

By Tricot's upper bound (1), both inequalities in (24) must, in fact, be equalities. Thus $\operatorname{dim}_{\mathrm{P}}(A)=\operatorname{dim}_{\mathrm{H}}(A \times B)-\operatorname{dim}_{\mathrm{H}}(B)$, as desired.

For analytic sets $A \subset \mathbf{R}^{d}$ with $\operatorname{dim}_{\mathrm{P}}(A)=d$, the variational formula (2) follows easily from the validity of this formula for any closed subset $A_{0}$ of $A$ with $d-\epsilon<$ $\operatorname{dim}_{\mathrm{P}}\left(A_{0}\right)<d$, by taking $\epsilon$ small. (The existence of such subsets $A_{0}$ is a consequence of the work of Joyce and Preiss [7].) By a more involved recursive argument, we can show the supremum is attained in this case also, but since including this argument would lengthen the paper, we omit it.

Remark. The first occurrence we know of the expression

$$
\sup _{B}\left\{\operatorname{dim}_{\mathrm{H}}(A \times B)-\operatorname{dim}_{\mathrm{H}}(B)\right\}
$$

is in the work of Kaufman [8].

\section{Packing dimension of products: Proof of Proposition 1.2}

We must show that any two compact sets $E$ and $F$ in $\mathbf{R}^{d}$ satisfy $\operatorname{dim}_{\mathrm{P}}(E \times F) \geq$ $\underline{\operatorname{dim}}_{\mathrm{P}}(E)+\operatorname{dim}_{\mathrm{P}}(F)$. Assume that

$$
\alpha<\underline{\operatorname{dim}}_{\mathrm{P}}(E) \text { and } \beta<\operatorname{dim}_{\mathrm{P}}(F) .
$$

Applying Lemma 2.1( $i i)$ to the boundary of the tree $\partial T(F)$, we obtain a subtree $T(\widetilde{F})$ with $\widetilde{F} \subset F$ such that for any vertex $w$ of the tree $T(\widetilde{F})$, we have

$$
\limsup _{n \rightarrow \infty} \frac{1}{n} \log _{2}\left|T_{n}(\widetilde{F}, w)\right|>\beta,
$$

where $\left|T_{n}(\widetilde{F}, w)\right|$ is the number of descendants $u$ of $w$ such that $|u|=n$. Similarly, by part (iii) of Lemma 2.1, there is a subset $\breve{E}$ of $E$ such that any vertex $v$ of $T(\breve{E})$ satisfies

$$
\liminf _{n \rightarrow \infty} \frac{1}{n} \log _{2}\left|T_{n}(\breve{E}, v)\right| \geq \alpha .
$$

Consider any vertex $(v, w)$ of the product tree $T(\breve{E}) \times T(\widetilde{F})$. For $n>|v|=|w|$ we have

$$
\left|T_{n}(\breve{E} \times \widetilde{F},(v, w))\right|=\left|T_{n}(\breve{E}, v)\right| \cdot\left|T_{n}(\widetilde{F}, w)\right|,
$$

so the asymptotics (27) and (26) imply that

$$
\limsup _{n \rightarrow \infty} \frac{1}{n} \log _{2}\left|T_{n}(\breve{E} \times \widetilde{F},(v, w))\right| \geq \alpha+\beta .
$$

Invoking part $(i)$ of Lemma 2.1, we obtain $\operatorname{dim}_{\mathrm{P}}(E \times F) \geq \alpha+\beta$. Since $\alpha$ and $\beta$ are only restricted by the assumption (25), this completes the proof of (7).

Now we construct a compact set in $[0,1]$ for which the inequality in $(7)$ is strict. For $i=0,1$, let $E_{i}$ be a compact set in $[0,1]$ such that every vertex at level $n$ of the tree $T\left(E_{i}\right)$ has

$$
\begin{cases}\text { one child } & \text { if }(4 k+2 i) ! \leq n<(4 k+2 i+1) \text { ! for some } k \geq 1 \\ \text { two children } & \text { otherwise. }\end{cases}
$$

See Figure 4. Loosely speaking, the sets $E_{0}$ and $E_{1}$ are large at most scales, and the scales where they are small are disjoint. More precisely, if $v$ is a vertex of $T\left(E_{0}\right)$ 


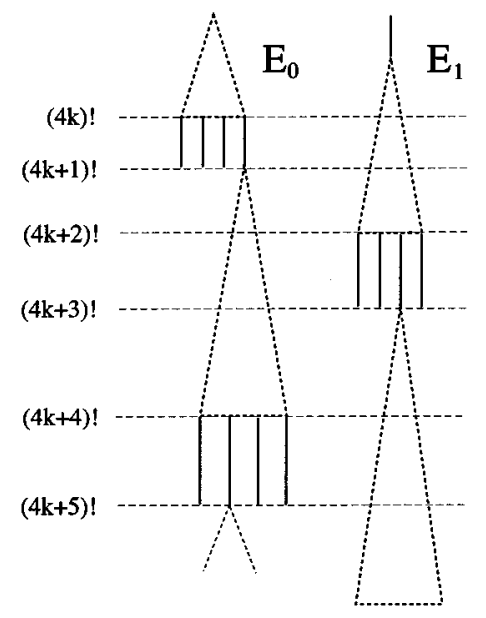

Figure 4. On disjoint scales, $E_{0}$ and $E_{1}$ have no branching.

with $|v| \leq(4 k+1)$ !, and if $n \in[(4 k+2) !$, $(4 k+4) !)$, then $\left|T_{n}\left(E_{0}, v\right)\right| \geq 2^{n-(4 k+1) !}$. Defining $S_{0}=\bigcup_{k=1}^{\infty}[(4 k+2) !,(4 k+4) !)$, we obtain

$$
\liminf _{n \in S_{0}} \frac{1}{n} \log _{2}\left|T_{n}\left(E_{0}, v\right)\right|=1 .
$$

for any vertex $v$ of $T\left(E_{0}\right)$. Similarly, defining $S_{1}=\bigcup_{k=1}^{\infty}[(4 k) !,(4 k+2) !)$, we get

$$
\liminf _{n \in S_{1}} \frac{1}{n} \log _{2}\left|T_{n}\left(E_{1}, v\right)\right|=1,
$$

Let $E=E_{0} \cup E_{1}$. We claim that

$$
\operatorname{dim}_{\mathrm{P}}(E \times F) \geq 1+\operatorname{dim}_{\mathrm{P}}(F) \text { for any compact set } F \subset[0,1] .
$$

(In fact this is an equality, but we only need one direction.)

To verify this claim, let $\beta<\operatorname{dim}_{\mathrm{P}}(F)$. As before, there is a tree $T(\widetilde{F})$ with $\widetilde{F} \subset F$ such that for any vertex $w$ of the tree $T(\widetilde{F})$, the asymptotic relation (26) holds. Now we distinguish two cases.

CASE I: All vertices $w$ of $T(\widetilde{F})$ satisfy

$$
\limsup _{n \in S_{0}} \frac{1}{n} \log _{2}\left|T_{n}(\widetilde{F}, w)\right|>\beta .
$$

In this case, $(28)$ implies that any vertex $(v, w)$ of the product tree $T\left(E_{0} \times \widetilde{F}\right)$ satisfies

$$
\limsup _{n \in S_{0}} \frac{1}{n} \log _{2}\left|T_{n}\left(E_{0} \times \widetilde{F}\right),(v, w)\right|>\beta .
$$

By Lemma 2.1(i), this yields $\operatorname{dim}_{\mathrm{P}}\left(E_{0} \times \widetilde{F}\right) \geq 1+\beta$, establishing the claim (30).

CASE II: There is a vertex $w$ of $T(\widetilde{F})$ that does not satisfy (31). In this case, by (26), every descendant $u$ of $w$ must satisfy 


$$
\limsup _{n \in S_{1}} \frac{1}{n} \log _{2}\left|T_{n}(\widetilde{F}, u)\right|>\beta,
$$

since the union $S_{0} \cup S_{1}$ contains all integers greater than 4 !. Let $T(\widetilde{F}, w)$ be the subtree of $T(\widetilde{F})$ which consists of all ancestors and descendants of $w$. Applying Lemma $2.1(i)$ to the boundary of $T\left(E_{1}\right) * T(\widetilde{F}, w)$ yields that $\operatorname{dim}_{\mathrm{P}}\left(E_{1} \times \widetilde{F}\right) \geq 1+\beta$, completing the proof of the claim (30).

Since clearly $\underline{\operatorname{dim}}_{\mathrm{M}}\left(E_{i}\right)=0$, for $i=0,1$, the definition of lower packing dimension gives $\operatorname{dim}_{\mathrm{P}}(E)=0$. Comparing this with the claim (30) shows that the inequality in Proposition 1.2 is strict for the set $E$ constructed here.

Remark. Hu and Taylor (1994) used the notation "aDim(E)" for

$$
\inf _{F}\left\{\operatorname{dim}_{\mathrm{P}}(E \times F)-\operatorname{dim}_{\mathrm{P}}(F)\right\} .
$$

It is easy to see that the sets $E_{0}, E_{1}$ in the preceding proof have $\operatorname{aDim}\left(E_{i}\right)=0$ for $i=0,1$, so the inequality $\operatorname{aDim}(E) \geq 1$ established above (which is really an equality) shows that the index aDim is not stable under finite unions.

Question. Theorem 1.1 expresses the packing dimension of a set $A$ in terms of Hausdorff dimensions. Is there an expression for the $\alpha$-dimensional packing measure of $A$ in terms of Hausdorff measures of sets associated to $A$ ?

Remark. After obtaining our results, we were informed by Professor S. J. Taylor that similar results were obtained independently by Dr. Yimin Xiao in the special case $d=1$. His paper will appear in Math. Proc. Camb. Phil. Soc.

\section{REFERENCES}

[1] Benjamini, I. and Peres, Y. (1992). Random walks on a tree and capacity in the interval. Annales Inst. Henri Poincaré, Probab. et Stat. 28, 557-592. MR 94f:60089

[2] Benjamini, I. and Peres, Y. (1994). Tree-indexed Random walks on groups and first passage percolation. Probab. Th. rel. Fields. 98, 91-112. MR 94m:60141

[3] Besicovitch, A.S., and Moran, P.A.P. (1945). The measure of product and cylinder sets. J. London Math. Soc. 20, 110-120. MR 8:18f

[4] Falconer, K.J. (1990). Fractal geometry: mathematical foundations and applications. John Wiley \& Sons. MR 92j:28008

[5] Falconer, K.J. and Howroyd, J. D. (1996). Projection theorems for Box and Packing dimension. To appear in Math. Proc. Camb. Phil. Soc. 119, 287-295. CMP 96:03

[6] Hu, X. and Taylor, S. J. (1994). Fractal properties of products and projections of measures in $\mathbf{R}^{d}$. Math. Proc. Camb. Phil. Soc. 115, 527-544. MR 95f: 28013

[7] Joyce, H. and Preiss, D. (1995). On the existence of subsets of finite positive packing measure. To appear in Mathematika 42, 1-15. CMP 95:17

[8] Kaufman, R. (1987). Entropy, dimension, and random sets. Quart. J. Math. Oxford 38, 77-80. MR 88d:28028

[9] Marstrand, J.M. (1954). Some fundamental geometric properties of plane sets of fractal dimension. Proc. London Math. Soc. 4, 257-302. MR 16:121g

[10] Mattila, P. (1995). Geometry of sets and measures in Euclidean space. Cambridge University Press. CMP 95:13

[11] Mattila, P. and Mauldin, R. D. (1994). Measure and dimension functions: measurability and densities. University of Jyväskylä Preprint 175.

[12] Taylor, S. J. (1986). The measure theory of random fractals. Math. Proc. Camb. Phil. Soc. 100, 383-406. MR 87k:60189

[13] Tricot, C. (1980). Rarefaction indices. Mathematika 27, 46-57. MR 81j:28005 
[14] Tricot, C. (1982). Two definitions of fractal dimension. Math. Proc. Camb. Phil. Soc. 91, 57-74. MR 84d:28013

[15] Tricot, C. (1991). Rectifiable and fractal sets. in Fractal Geometry and Analysis, NATO ASI Series C: v. 346, pp. 532-541, Edited by J. Bélair and S. Dubuc, Kluwer Academic Publishers.

Department of Mathematics, SUny at Stony Brook, Stony Brook, New York 117943651

E-mail address: bishop@math.sunysb.edu

Department of Statistics, University of California, Berkeley, California 94720

Current address: Institute of Mathematics, The Hebrew University, Givat Ram, Jerusalem 91904, Israel

E-mail address: peres@math.huji.ac.il 